\title{
Contrôle de la phase relative d'impulsions harmoniques à une échelle de temps attoseconde
}

\author{
J.-F. Hergott, M. Kovacev, F. Vernay, E. Priori ${ }^{1}$, H. Merdji, P. Salières et B. Carré \\ Service des Photons, Atomes et Molécules, CEA-Saclay, 91191 Gif-sur-Yvette, France \\ ${ }^{1}$ INFM, Dipartimento di Fisica, Politecnico, piazza Leonardo da Vinci 32, 20133 Milano, Italie
}

\begin{abstract}
Résumé : Nous démontrons la possibilité de générer quatre impulsions harmoniques bloquées en phase séparées temporellement, que nous caractérisons par interférométrie fréquentielle. Les spectres présentent alors une sensibilité extrême au déphasage entre les deux couples d'impulsions qui interfèrent. Nous montrons qu'un contrôle de cette phase relative peut être fait à une échelle attoseconde (1as $=10^{-18} \mathrm{~s}$ ). La résolution spectrale et la haute sensibilité de cette technique pourraient être utilisées pour des mesures de haute précision en spectroscopie et interférométrie.
\end{abstract}

\section{INTRODUCTION}

La source UVX par génération d'harmoniques d'ordre élevé (GHOE) présente des propriétés uniques de courte durée et de cohérence élevée. Elle est devenue une source privilégiée pour de nombreuses applications. La courte durée des harmoniques a été caractérisée par différentes méthodes indiquant une durée de quelques femtosecondes selon la durée de l'impulsion laser de pompe [1-3]. Cette propriété est primordiale pour des applications de type pompe sonde comme par exemple en spectroscopie moléculaire et atomique [4] ou encore en physique du solide [5]. Le degré de cohérence intrinsèque élevé a également été démontré, aussi bien temporellement [6,7] que spatialement [8,9]. D'autre part, il a été démontré récemment la possibilité de produire deux sources harmoniques séparées dans l'espace mais mutuellement cohérentes [10]. Les deux faisceaux interfèrent alors en champ lointain après propagation et recouvrement spatial. Cette technique originale nous a permis de réaliser une cartographie 2-D de la densité électronique d'un plasma produit par laser [11]. L'analogue temporel de cette technique, qui consiste à produire deux impulsions harmoniques séparées en temps mais bloquées en phase a également été démontré $[12,13]$, et utilisé pour le diagnostic de plasmas par interférométrie fréquentielle UVX $[12,14]$.

Nous exposons dans cet article une extension de cette méthode à quatre impulsions. Après une description de l'interféromètre, nous présentons les résultats expérimentaux démontrant l'extrême sensibilité de la technique.

\section{RESULTATS EXPERIMENTAUX}

Ces expériences ont été réalisées sur le serveur laser LUCA du DRECAM à Saclay. Ce système délivre des impulsions infra rouge (IR) à $800 \mathrm{~nm}$, d'énergie allant jusqu'à $100 \mathrm{~mJ}$, de durée $50 \mathrm{fs}$ à un taux de répétition de $20 \mathrm{~Hz}$. Les quatre impulsions harmoniques bloquées en phase sont générées en produisant quatre impulsions laser séparées en temps, focalisées dans un jet de gaz comme illustré en Figure 1. Un premier couple d'impulsions est produit en utilisant la différence de vitesse de groupe sur les deux axes d'une lame biréfringente tournée à $45^{\circ}$ par rapport à la polarisation du laser. L'épaisseur calibrée de la lame fixe le délai $\tau$ entre les deux impulsions à $120 \mathrm{fs}$. Un polariseur, placé après cette dernière, projette les deux composantes sur le même axe. Le couple est envoyé dans un interféromètre de type Michelson où deux répliques $\left(\mathrm{C}_{1}\right.$ et $\mathrm{C}_{2}$ ) sont produites. La longueur du premier bras de l'interféromètre est contrôlée par un moteur pas à pas, 
tandis que le second bras est ajusté finement par une translation piézoélectrique permettant une précision nanométrique. De plus, des mesures interférométriques ont confirmé une stabilité de ce dispositif de l'ordre de $10 \mathrm{~nm}$. Ceci nous permet de contrôler très précisément les chemins optiques des deux couples $C_{1}$ et $C_{2}$ de l'impulsion laser et ainsi le délai temporel $\Delta \mathrm{T}$ entre les deux couples. Ces quatre impulsions IR sont focalisées dans un jet de gaz (xénon) où elles produisent quatre impulsions harmoniques qui sont envoyées dans un spectromètre constitué d'un miroir torique et d'un réseau plan. La dispersion spectrale permet aux impulsions d'interférer et de produire un spectre cannelé qui est enregistré par des galettes de microcanaux (MCP) placées dans le plan focal du spectromètre. Celles-ci sont montées en incidence rasante $\left(12^{\circ}\right)$ afin d'augmenter la résolution effective à $0.1 \AA$.

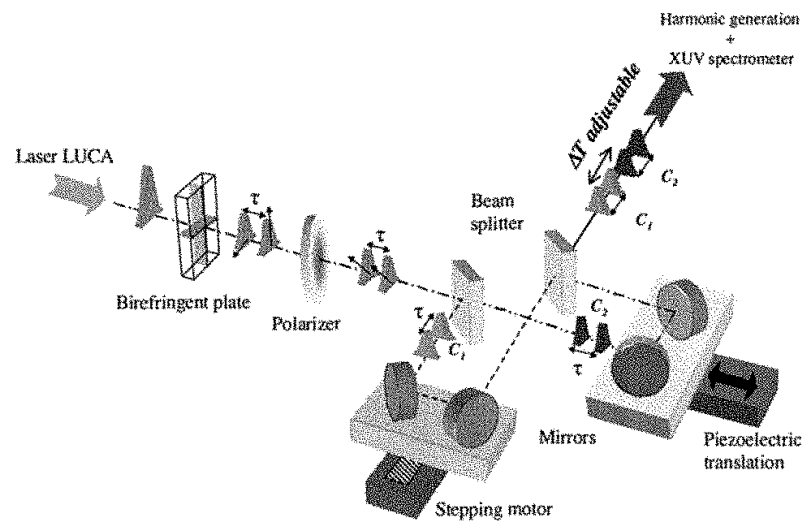

Figure 1. Schéma du dispositif expérimental utilisé pour la génération de quatre impulsions laser décalées en temps.

Lorsque les deux bras de l'interféromètre ne sont pas alignés les deux couples ne sont pas superposés spatialement, induisant deux systèmes de franges correspondant chacun à l'interférence spectrale des deux impulsions constituant chaque couple, avec la même période de franges fixée par le délai $\tau: \Delta \lambda=\lambda^{2} / c \tau$ (Figure 2a). Les systèmes de franges observés sont indépendants du délai $\Delta T$ entre les couples $C_{1}$ et $C_{2}$.
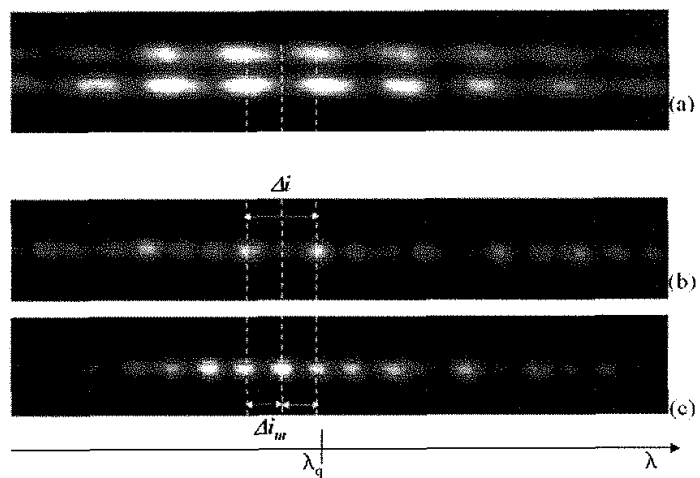

Figure 2. Spectre de puissance pour la $11^{\text {ème }}$ harmonique générée dans le xénon résultant de 1'interférence des deux couples d'impulsions décalées en temps ( $\tau=120 \mathrm{fs})$. (a) L'interféromètre est désaligné spatialement . (b) et (c) L'interféromètre est aligné spatialement et le décalage $\Delta T$ entre les deux couples est respectivement $2 \tau$ (b) et $2 \tau+T_{\mathrm{t}} / 2$ (c). 
Ces spectres sont obtenus en un seul tir. La stabilité du dispositif expérimental permet cependant une accumulation sur un grand nombre de tirs avec une dégradation limitée du contraste de franges. Un réalignement de l'interféromètre permet une superposition spatiale des couples d'impulsions harmoniques. Si les deux couples n'étaient pas mutuellement cohérents, la superposition spatiale se résumerait à une somme des deux interférogrammes; la période des franges reste la même et l'intensité est doublée. En fait, nous observons un changement important du système de franges, comme illustré en Figure $2 \mathrm{~b}$. Ceci démontre la génération de quatre impulsions harmoniques bloquées en phase et séparées en temps. Le retard $\Delta T$ entre $C_{1}$ et $\mathrm{C}_{2}$ était fixé à $2 \tau$ correspondant à quatre impulsions régulièrement espacées de $\tau$. Un clair affinement des franges est ainsi obtenu (d'un facteur 2 par rapport à deux impulsions). En ajustant finement le retard à $\Delta \mathrm{T}=2 \tau+\mathrm{T}_{q} / 2$ (où $\mathrm{T}_{\mathrm{q}} / 2$ est la demi période harmonique, $\sim 0.12 \mathrm{fs}$ pour la $11^{\text {ème }}$ harmonique, ce qui correspond à un déplacement de $18 \mathrm{~nm}$ des miroirs d'un bras du Michelson), nous observons un net changement du système de franges, qui devient plus régulier avec une période de franges deux fois plus petit (figure 2c). Nous avons choisi des conditions de la génération $\left(50 \mathrm{torr} / 5 \times 10^{13} \mathrm{~W} / \mathrm{cm}^{2}\right)$ permettant d'avoir une "faible ionisation" du milieu générateur et ainsi obtenir des profils spectraux très réguliers.

En Figure 3, nous montrons les profils spectraux correspondant aux images enregistrées. Dans le cas d'une seule double impulsion, la modulation du spectre harmonique présente un interfrange de $1,1 \AA$ et la largeur à mi-hauteur de chaque frange est $\mathrm{L}_{2}=0,65 \AA$. Dans le cas de deux couples mutuellement cohérents qui interfèrent avec un délai $\Delta \mathrm{T} \sim 2 \tau$ la période reste la même $(\Delta \mathrm{i}=1,1 \AA)$ alors que la largeur à mi-hauteur du pic principal, entouré par des pics satellites, est divisée par $2 ; L_{4}=1 / 2 L_{2} \sim 0,3 \AA$. D'autre part, lorsque $\Delta \mathrm{T} \sim 2 \tau+\mathrm{T}_{\mathrm{q}} / 2$, la période des franges diminue de moitié $\left(\Delta \mathrm{i}_{\mathrm{m}}=0,5 \AA\right)$ avec des pics réguliers alors que la largeur à mi-hauteur reste pour sa part identique au cas précédent.

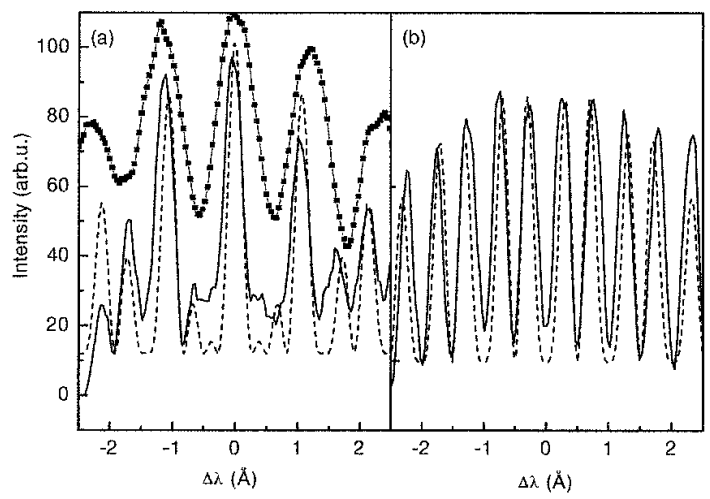

Figure 3. Spectres de puissance (trait plein) résultant de l'interférence de deux couples d'impulsions (H11) décalées en temps de $2 \tau$ (a) et $2 \tau+\mathrm{T}_{\mathrm{q}} / 2$ (b). En trait pointillé sont présentés les fits effectués avec la formule théorique. Le figure (a) montre aussi le spectre de puissance obtenu dans le cas d'une seule double impulsion (carrés).

Le contraste des franges est important dans chacun de ces deux cas, respectivement $80 \%$ et $60 \%$. Pour mieux comprendre cette évolution, il suffit d'écrire l'expression mathématique du spectre de puissance pour quatre impulsions bloquées en phase:

$$
\widetilde{I}(\omega)=\widetilde{I}_{0}(\omega) \times 16 \cos ^{2}\left(\frac{\omega \Delta T}{2}\right) \cos ^{2}\left(\frac{\omega \tau}{2}\right)
$$

Une modulation supplémentaire en $\cos ^{2}\left(\frac{\omega \Delta T}{2}\right)$ est introduite par rapport à une seule double impulsion. Ainsi, lorsque $\Delta \mathrm{T} \sim 2 \tau$, la périodicité des franges est conservée mais la largeur de ces dernières est diminuée. 
Lorsque $\Delta \mathrm{T} \sim 2 \tau+\mathrm{T}_{\mathrm{q}} / 2$, cette modulation est équivalente à un $\sin ^{2}\left(\frac{\omega \Delta T}{2}\right)$, qui n'oscille pas en phase avec la modulation lente. Ceci explique le dédoublement observé des pics principaux. Cette interprétation est confirmée par les fits dérivées de la formule théorique (1) (traits pointillés en Figure 3).

\section{CONCLUSION}

En conclusion, nous avons démontré la génération de quatre impulsions harmoniques bloquées en phase et séparées en temps. Les profils spectraux obtenus par interférométrie fréquentielle présentent une sensibilité élevée à un changement de la phase relative à une échelle de temps attoseconde, indiquant qu'un tel contrôle est réalisé sur l'émission harmonique. En effet, un déphasage d'une demi période harmonique $(\sim 0.12$ fs pour H11) résulte en une modification nette du système de franges. Notons que dans le cas d'une seule double impulsion, le déphasage se traduit uniquement par un décalage global du système de franges dans l'enveloppe spectrale. Des perspectives intéressantes résultent de l'application de cette technique. La haute sensibilité pourrait être utile pour le diagnostic précis des plasmas denses par interférométrie. L'affinement des franges associé à l'augmentation de l'intensité spectrale (d'un facteur 16 par rapport à une seule impulsion) permettraient des expériences de spectroscopie fine de type Ramsey dans l'UVX. Finalement, les expériences pompe sonde avec deux couples harmoniques séparés dans l'espace (Figure 2a) tireraient bénéfice de cette configuration qui offre la possibilité d'une référence absolue dans l'image.

\section{Références}

[1] Glover T.E., Schoenlein R.W., Chin A.H. et Shank C.V., Phys. Rev. Lett. 76 (1996) 2468; J.M. Schins et al. J. Opt. Soc. Am. B 13 (1996) 197

[2] Toma E.S., Muller H.G., Paul P.M., Breger P., Cheret M., Agostini P., Le Blanc C., Mullot G. et Cheriaux G., Phys. Rev. A 62 (2000) 061801

[3] Drescher M., Hentschel M., Kienberger R., Tempea G., Spielmann C., Reider G.A., Corkum P.B. et Krausz F., Science 291 (2001) 1923

[4] Larsson J., Mevel E., Zerne R., L'Huillier A., Wahlström C.-G. Svanberg S., J. Phys. B 28 (1995) L53

[5] Quéré F., Guizard S., Petite G., Martin Ph., Merdji H., Carré B. et. Hergott J.-F, Phys. Rev. B 61 (2000) 15

[6] Bellini M., Lyngå C., Tozzi A., Gaarde, M. B., Delfin C., Hänsch T.W., L' Huillier A. et Wahlström C.G., Phys. Rev. Lett. 81 (1998) 297

[7] Lyngå C., Gaarde, M. B., Delfin C., Bellini M., Hänsch T.W., L' Huillier A. et Wahlström C.-G., Phys. Rev. A 60 (1999) 4823

[8] Ditmire T., Grumbell E.T., Smith R.A., Tisch J.W.G., Meyerhofer D.D. et Hutchinson M.H.R., Phys. Rev. Lett. 77 (1996) 4756

[9] LeDéroff L., Salières P., Carré B., Joyeux D. et Phalippou D., Phys. Rev. A 61 (2000) 043802

[10] Zerne R., Altucci C., Bellini M., Gaarde M.B., Hänsch T.W., L'Huillier A., Lyngå C. et Wahlström C.G., Phys. Rev. Lett. 79 (1997) 1006

[11] Descamps D., Lyngå C., Norin J., L'Huillier A., Wahlström C.-G., Hergott J.-F., Merdji H., Salières P., Bellini M., et Hänsch T. W., Opt. Lett 25 (2000) 135

[12] Salières P., LeDéroff L., Auguste T., Monot P., d'Oliveira P., Campo D., Hergott J.-F., Merdji H. et Carré B., Phys. Rev. Lett. 83 (1999) 5483

[13] Hergott J.-F., Salières P., Merdji H., LeDéroff L., Carré B., Auguste T., Monot P., d'Oliveira P., Descamps D., Lyngå C., Norin J., L'Huillier A., Wahlström C.G., Bellini M. et Hänsch T.W., Las. Part. Beams 19 (2000)

[14] Hergott J.-F., Auguste T., Salières P., Le Déroff L., Monot P., d'Oliveira P., Campo D., Merdji H. et B. Carré, soumis à J. Opt. Soc. Am. B (2002) 